\title{
Acute health events in adult patients with genetic disorders: The Marshfield Epidemiologic Study Area
}

Philip F. Giampietro, $M D, P h D^{1}$, Robert T. Greenlee, $P h D, M P H^{2}$, Elizabeth McPherson, $M D^{1}$, Lorelle L. Benetti, AS $S^{3}$, Richard L. Berg, $M S^{3}$, and Stephen F. Wagner, $M D^{1}$

\begin{abstract}
Purpose: We ascertained and reviewed acute health events occurring in 2003 among patients age 18 and greater with well-defined single gene, chromosomal, and selected multifactorial conditions within the Marshfield Epidemiologic Study Area. Methods: Of 47,077 adult Marshfield Epidemiologic Study Area Central cohort members, 1831 (3.9\%) had been given at least one of 71 ICD-9 codes appropriate for genetic diagnoses of interest. Physician review narrowed this to 591 (1.3\%) validated patients for the study. Of the 591,527 (89.2\%) patients registered 6,849 visits, which were manually reviewed to delineate acute, relevant health events in the urgent care and primary care provider setting. Results: A total of 244 acute relevant health events among 126 patients corresponding to 58 different genetic conditions were observed. Acute relevant health events corresponded to $3.4 \%$ of the total health events in patients identified with genetic problems. Categories of genetic conditions with the highest frequencies of acute relevant health events included chromosomal and microdeletion syndromes (21.3\%), hematologic disorders (11.5\%), muscular dystrophies (8.6\%), and connective tissue disorders (10.2\%). Conclusions: These data have multiple applications and implications in addressing the natural history, long-term medical needs and financial impact of adult patients with genetic conditions. Genet Med 2006:8(8):474-490.
\end{abstract}

Key Words: adult genetic disease, education, long-term follow-up data, MESA population, natural history

Patients with genetic conditions constitute an important portion of the world population with special health care needs. Estimates indicate an incidence of 3.8 per 1,000 for chromosomal disorders, 20 per 1,000 for single gene disorders, and 646.4 per 1,000 for multifactorial disorders. ${ }^{1}$ Previous studies during the past three decades have quantified the frequency of genetic disorders among patients especially children, admitted to the hospital..$^{-9}$ In general patients with genetic problems have more admissions, longer hospital stays, and increased morbidity and mortality. ${ }^{1-3,9}$ These studies have not addressed specific reasons relating to a given diagnosis which led to hospital admission.

A patient with an underlying genetic disorder may present to an urgent care setting or health provider's office with onset of acute symptoms that require immediate attention and/or further evaluation. A prior study ${ }^{10}$ described pediatric patients with known genetic conditions presenting to an emergency department (ED) in a municipal hospital. That study reviewed paper records obtained from ED visits and relied on reporting of genetic diagnoses in the ED encounter form filled out by the

\footnotetext{
From the ${ }^{1}$ Department of Medical Genetics, Marshfield Clinic, Marshfield, Wisconsin; ${ }^{2}$ Marshfield Epidemiology Research Center; and ${ }^{3}$ Biostatistics and Bioinformatics Core, Marshfield Clinic Research Foundation, Marshfield, Wisconsin.

Philip F. Giampietro, Department of Medical Genetics, Marshfield Clinic, 1000 North Oak Avenue, Marshfield, WI 54449.

Submitted for publication March 20, 2006.
}

Accepted for publication June 1, 2006.

DOI: 10.1097/01.gim.0000232479.90268.40
ED physician. There was no linkage of the ED encounter with the patient's hospital chart which would be important in the inclusion of/or verifying the genetic diagnosis. To date, the burden of genetic disorders among adults in ambulatory settings remains poorly characterized.

The study described in this report delineates acute health events identified in the ED, urgent care, and primary care setting among a cohort of 527 adult patients with single gene, chromosomal, and syndromic conditions within a geographically defined, population-based cohort. A goal of the study was to initiate a means of collecting data about the ongoing health effects of genetic diseases among adults in a general population. It was expected that such information could provide insight into the natural history of adult genetic conditions and improve transitional care for genetic diseases. It could be used to help foster physician, patient, and family education.

\section{METHODS}

This study was conducted in the Marshfield Epidemiologic Study Area (MESA), ${ }^{11}$ a resource for population-based surveillance and research within the context of an integrated health care delivery system. Covering a region where nearly all residents receive their care from the Marshfield Clinic and affiliated hospitals, MESA has been particularly useful for quantifying the occurrence of illness and studying the full clinical spectrum of disease in a general population. ${ }^{12}$ MESA is divided into two main subareas, MESA Central which contains about 54,000 people in 14 ZIP codes surrounding the Clinic's main 
campus in the city of Marshfield, and MESA North which contains about 23,000 people in 10 ZIP codes surrounding several of the Clinic's regional centers in northern Wisconsin. The main campus of the Clinic is adjacent to St. Joseph's Hospital in Marshfield, the only major rural referral medical center in Wisconsin. Marshfield Clinic physicians staff the hospital and the two institutions share a common electronic medical record system. MESA Central was selected for this project because of the greater utilization of emergent care at the main campus in Marshfield by MESA Central residents.

Individual residency status and follow-up time have been tracked for the cohort since 1991. Research is initiated through linking MESA residency data with the Marshfield Clinic's extensive archive of medical information, which includes a full electronic medical record dating back to the early 1990s and a file of more than 100 million patient diagnoses dating back to 1960. Mobility in the MESA region is quite low, resulting in greater capture of a patient's full medical history in the Clinic's medical records. Only about 3\% per year of the original 1991 cohort is lost to out-migration.

The high population and health event coverage in MESA are validated periodically through random sample surveys and comparisons with state and national data sources. The most recent validation indicates that Marshfield Clinic data systems capture more than $97 \%$ of the residents, $99 \%$ of the deaths, $95 \%$ of hospital discharges, and $90 \%$ of outpatient visits in MESA Central. ${ }^{12}$

Institutional Review Board approval for the study was obtained. Identifiable data from the electronic study files were removed after the data were collected. Data collection for this study occurred in two stages, which is outlined in Figure 1. First, adults currently residing in the MESA Central region were screened for past evidence of selected underlying genetic conditions using ICD-9 diagnostic codes, and the medical records of those potentially affected were reviewed in order to validate and properly classify their underlying conditions. Second, the cohort of adults with confirmed genetic conditions were followed historically over the course of calendar year 2003, and all ED, urgent care, and primary care visits were catalogued for further review by clinical geneticists (PFG, EM, SFW) to identify and classify acute, relevant health events.

For the first stage of data collection, potential underlying genetic diagnoses under consideration were classified based on Hall's study ${ }^{5}$ as follows: Group A) multifactorial conditions (i.e., hypercholesterolemia), Group B) conditions that are suggestive of an underlying genetic diagnosis but not proven (i.e., hearing loss), and Group C) well-defined single gene, chromosomal or multifactorial conditions (e.g., cystic fibrosis, Down syndrome, spina bifida).

We chose to focus primarily on group $\mathrm{C}$ conditions in order to delineate acute health events occurring among patients with well-defined, single gene, chromosomal or selected multifactorial conditions such as spina bifida and Hirschsprung disease. We suspected that these conditions could have long-term sequelae, which could be associated with acute health events in adulthood. Following an in-depth review of the ICD-9 coding
47,077 adult patients in MESA Central during 2003

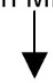

Apply 71 ICD 9 codes inclusive for underlying genetic diagnoses



1,831 patients with at least 1 selected

ICD-9 diagnosis at any point since 1979

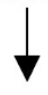

Manual review

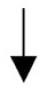

610 charts with underlying genetic conditions validated

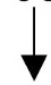

591 unique patients in cohort



64 patients with no emergency room/urgent care or other outpatient events in 2003

527 unique patients with one or more emergency room/ urgent care or other outpatient visits in 2003

Fig. 1. Flow diagram illustrating ascertainment of 527 adult patients with underlying genetic conditions in MESA in 2003.

manual and consultation with the Clinic's health information management coders, a broad array of 71 ICD-9 diagnostic code groupings was selected to enhance our capture of adults who may have clinically recognized genetic conditions of interest. These ICD9 codes included more generalized conditions, such as "conduction disorders-426" and "hereditary and degenerative diseases of the central nervous system-330-337," as well as specific genetic conditions, such as "polycystic kidney disease753" and "cystic fibrosis-277."

The Clinic's data warehouse archive was queried to identify any instances of the 71 selected diagnostic codes being given since 1979 to the 47,077 adult members of the MESA Central cohort as of January 1, 2003. Validation review of the corresponding medical charts applied established clinical criteria to determine whether patients classified in a particular diagnostic category likely had the conditions of interest. The majority of the charts were reviewed by clinical geneticists (PFG, EM, SFW), although charts for a small number of conditions (disorders of iron, magnesium, phosphorous, and calcium metabolism, and hemoglobinopathies, not elsewhere classified) were prescreened by trained medical abstractors to first determine whether an identified Mendelian disease may be present (e.g., hemochromatosis, Hallervorden-Spatz, Aceruloplasminemia and Friedreich ataxia). Review of the paper chart was necessary for approximately $37.5 \%$ of the diagnoses when validation of a patient's possible genetic diagnosis was not possible with the electronic record. In some instances review of X-rays was performed in order to clarify the diagnosis of a skeletal dysplasia. 


\section{Giampietro et al.}

Ten percent of the records were randomly selected for independent review by a separate geneticist co-investigator in order to ensure the accuracy of the final genetic diagnoses. The geneticists met periodically to collectively review the genetic diagnoses that were assigned and adjudicate ambiguous cases. A corresponding Online Mendelian Inheritance in Man (OMIM) code was assigned to each genetic diagnosis, when applicable, in order to facilitate database management. Diagnosis validity and final classifications were recorded by the reviewers on paper abstraction forms and entered into an electronic database by trained staff using standard double data entry procedures to reduce entry errors.

In the second stage of data collection, the patients with confirmed genetic conditions identified in stage 1 were assessed for presence of ED, urgent care, or primary care visits during calendar year 2003. Those with visits in 2003 underwent medical record review to: 1 ) establish the specific diagnoses given in the encounter, 2) determine whether each diagnosis was pathogenically relevant to the underlying genetic condition, and 3) assess whether the event was acute or not. Relevance was ascertained by means of verification of associated complications in standard clinical genetics reference books and Internet resources (e.g., "Smith's Recognizable Patterns of Human Malformation," 13 "Emery and Rimoin's Principles and Practice of Medical Genetics," "The Metabolic and Molecular Basis of Inherited Disease," 14 Online Mendelian Inheritance in Man (OMIM), ${ }^{15}$ Gene Clinics ${ }^{16}$ ) and literature review. Acute events were defined as those which required urgent scheduling of a diagnostic evaluation, immediate pharmacologic, antibiotic, or surgical intervention, or hospitalization for treatment. Acute health events were those that stood apart from routine care or monitoring that would occur during a general practice visit. The health visits associated with each underlying genetic diagnosis category were assigned to one of three clinical geneticists (PFG, EM, SFW). In addition to acuity and relevance, abstraction forms captured information on whether the health event required hospitalization. Also, the reviewing geneticist assigned relevant events to the appropriate organ system affected. Attempts were made to classify the organ system involved based on the known pathogenesis of a given condition. For example, one patient with achondroplasia and clinical symptoms of a progressive myelopathy, including paresthesias and loss of voiding sensation, was ultimately admitted for a C7-T1 discectomy and fusion for a herniated disc. Since the pathologic etiology for this patient's acute and relevant problem was in the spinal column, the acute and relevant events were classified under the "musculoskeletal" heading. Another patient with Down syndrome and Eisenmenger syndrome was diagnosed as having secondary polycythemia. This was included under the cardiovascular system, since the polycythemia was secondary to her underlying congenital heart disease. Meetings between the clinical geneticists were held regularly to review health events and discuss classification of acuity, relevance, and organ system classification.

The health event review data were then appended to the study data file using double data entry procedures. Analysis of the resulting data were descriptive, with summary tabulation and careful consideration of result implications.

\section{RESULTS}

We identified that 1831 (3.9\%) of the 47,077 MESA Central adults had been given at least one ICD-9 code since 1979 within the 71 selected code ranges appropriate for the genetic diagnoses of interest. Validation review narrowed this to 591 patients $(1.3 \%)$ with confirmed genetic conditions for further study (Fig. 1). Of those, 64 (10.8\%) registered no outpatient health events in 2003 resulting in 527, corresponding to $1.2 \%$ of the total adult patients in MESA. Among the remaining 527, 7139 visits were distributed between $\mathrm{ED}$, urgent care, and other primary care departments (mean of 13.0 visits per patient; median number of 9 visits per patient). This was $1.8 \%$ of the total outpatient visits in 2003 among the adult MESA Central cohort. These visits resulted in 19,288 diagnosis records (mean 2.8 diagnoses per patient per visit) and 1,702 unique conditions diagnosed.

Table 1 displays the selected genetic diagnoses and corresponding OMIM codes within categories generally defined by the affected organ system. For each underlying diagnosis, the number of affected patients within the cohort, the number of health events occurring in 2003, and the number and proportion of those events determined to be acute and relevant to their underlying diagnosis are shown. Sixteen patients had more than one underlying genetic diagnosis, and so are represented in the table more than once. A total of 244 acute relevant health events occurred among 126 patients, corresponding to 58 different genetic conditions. Of the acute relevant events, $190(77.9 \%)$ occurred in the urgent care/ED setting and 54 $(22.1 \%)$ occurred in the primary care or specialty provider's office. Acute relevant health events accounted for 3.6\% of the total health events among patients identified with genetic problems. Overall, nearly 1 in 4 patients in the cohort who had an outpatient visit in 2003 (126/543) presented with at least one acute health event that was relevant to their underlying genetic diagnosis.

Within the studied cohort, chromosomal and microdeletion syndromes accounted for about $11 \%$ of the underlying genetic conditions and a similar proportion (9\%) of the total outpatient visits in 2003, but comprised the greatest percentage $(21.3 \%)$ of the acute and relevant health events. This group was largely represented by patients with Down syndrome and sex chromosome abnormalities. A very high proportion (43.1\%) of patients with chromosome abnormalities had at least one acute relevant event during the year, suggesting that patients within this category were at a relatively high risk for an acute health event. Other genetic disease categories observed to have a high risk of acute, relevant health events were immunologic conditions (65\%), well-known non-chromosomal conditions, including Klippel-Trenaunay-Weber syndrome (44\%), muscular dystrophy (42\%), and genodermatoses (35\%). Hematologic disorders made up the largest group of patients in the study cohort $(21.7 \%)$, mostly represented by Factor V Leiden and von Willebrand disease, and contributed a similar proportion of total outpatient visits (24.4\%). They also accounted for a sizable proportion of total acute relevant events (11.5\%), and 
Table 1

Genetic diagnoses and health events for 2003

\begin{tabular}{|c|c|c|c|c|c|c|}
\hline $\begin{array}{l}\text { Category of } \\
\text { diagnosis }\end{array}$ & Genetic diagnosis & OMIM code & $\begin{array}{l}\text { Number of patients } \\
\quad(\% \text { of total })\end{array}$ & $\begin{array}{l}\text { Number of events } \\
\text { among patients } \\
\text { (\% of total) }\end{array}$ & $\begin{array}{l}\text { Number of acute/ } \\
\text { relevant events } \\
\text { (\% of total) }\end{array}$ & $\begin{array}{l}\text { Total patients with } \\
1 \text { or more acute/ } \\
\text { relevant events }\end{array}$ \\
\hline \multirow{2}{*}{$\begin{array}{l}\text { Cardiac } \\
\text { arrhythmia } \\
\text { syndromes }\end{array}$} & & & $1(0.18)$ & $1(0.01 \%)$ & $\mathbf{0}$ & 0 \\
\hline & Long QT syndrome 1 & 192500 & $1(0.18)$ & 1 & 0 & 0 \\
\hline \multirow{8}{*}{$\begin{array}{l}\text { Chromosomal and } \\
\text { microdeletion } \\
\text { syndromes }\end{array}$} & & & $58(10.68 \%)$ & $643(9.0 \%)$ & $52(21.3 \%)$ & $25(43.1 \%)$ \\
\hline & Angelman syndrome & 105830 & $1(0.18)$ & 11 & 1 & 1 \\
\hline & Prader-Willi syndrome & 176270 & $2(0.37)$ & 34 & 2 & 1 \\
\hline & Velocardiofacial syndrome & 192430 & $1(0.18)$ & 7 & 2 & 1 \\
\hline & Williams-Beuren syndrome & 194050 & $1(0.18)$ & 2 & 0 & 0 \\
\hline & Down syndrome & & $32(5.89)$ & 354 & 34 & 16 \\
\hline & $\begin{array}{l}\text { Sex chromosome (Klinefelter, } \\
\text { Turner, XX male) }\end{array}$ & & $18(3.31)$ & 187 & 11 & 5 \\
\hline & $\begin{array}{l}\text { Other chromosomal abnormality } \\
\text { (3p monosomy, Del 5q, } \\
\text { marker chromosome 15) }\end{array}$ & & $3(0.54)$ & 48 & 2 & 1 \\
\hline \multirow{10}{*}{$\begin{array}{l}\text { Connective tissue } \\
\text { disorders }\end{array}$} & & & $31(5.71 \%)$ & $578(8.1 \%)$ & $25(10.2 \%)$ & $8(25.8 \%)$ \\
\hline & Stickler syndrome, type I & 108300 & $2(0.37)$ & 8 & 2 & 1 \\
\hline & Dupuytren contracture & 126900 & $1(0.18)$ & 34 & 0 & 0 \\
\hline & Ehlers-Danlos syndrome, type III & 130020 & $1(0.18)$ & 108 & 9 & 1 \\
\hline & Ehlers-Danlos syndrome, type IV & 130050 & $1(0.18)$ & 18 & 0 & 0 \\
\hline & Marfan syndrome & 154700 & $12(2.21)$ & 226 & 6 & 3 \\
\hline & Mitral valve prolapse, familial & 157700 & $1(0.18)$ & 38 & 0 & 0 \\
\hline & $\begin{array}{l}\text { Pseudoxanthoma elasticum } \\
\text { dominant/recessive }\end{array}$ & $\begin{array}{c}177850 / \\
264800\end{array}$ & $2(0.36)$ & 16 & 2 & 1 \\
\hline & MASS syndrome & 604308 & $9(1.66)$ & 97 & 1 & 1 \\
\hline & $\begin{array}{l}\text { Connective tissue disorder } \\
\text { unspecified }\end{array}$ & & $2(0.36)$ & 33 & 5 & 1 \\
\hline \multirow[t]{4}{*}{ Dermatologic } & & & $14(2.58 \%)$ & $180(2.5 \%)$ & $3(1.2 \%)$ & $2(14.3 \%)$ \\
\hline & $\begin{array}{l}\text { Ichthyosis (vulgaris, lamellar, } \\
\text { X-linked) }\end{array}$ & $\begin{array}{c}146700 / \\
146750 / \\
242300 / \\
308100\end{array}$ & $12(2.21)$ & 173 & 3 & 2 \\
\hline & Cutis Laxa, X-linked & 304150 & $1(0.18)$ & 6 & 0 & 0 \\
\hline & Incontinentia pigmenti & 308300 & $1(0.18)$ & 1 & 0 & 0 \\
\hline \multirow[t]{4}{*}{ Ear, nose, throat } & & & $4(0.74 \%)$ & $51(0.7 \%)$ & $1(0.4 \%)$ & $1(25 \%)$ \\
\hline & Choanal atresia, posterior & 214800 & $2(0.37)$ & 3 & 0 & 0 \\
\hline & Pendred syndrome & 274600 & $1(0.18)$ & 30 & 1 & 1 \\
\hline & Usher syndrome, type IA & 276900 & $1(0.18)$ & 18 & 0 & 0 \\
\hline \multirow[t]{3}{*}{ Eye } & & & $66(12.15 \%)$ & $734(10.3 \%)$ & $8(3.3 \%)$ & $3(4.5 \%)$ \\
\hline & Blepharochalasis, superior & 110000 & $1(0.18)$ & 4 & 0 & 0 \\
\hline & $\begin{array}{l}\text { Corneal dystrophy, epithelial } \\
\text { basement membrane }\end{array}$ & 121820 & $1(0.18)$ & 1 & 0 & 0 \\
\hline
\end{tabular}


Table 1

Genetic diagnoses and health events for 2003, continued

\begin{tabular}{|c|c|c|c|c|c|c|}
\hline $\begin{array}{l}\text { Category of } \\
\text { diagnosis }\end{array}$ & Genetic diagnosis & OMIM code & $\begin{array}{l}\text { Number of patients } \\
\text { (\% of total) }\end{array}$ & $\begin{array}{l}\text { Number of events } \\
\text { among patients } \\
\text { (\% of total) }\end{array}$ & $\begin{array}{l}\text { Number of acute/ } \\
\text { relevant events } \\
\text { (\% of total) }\end{array}$ & $\begin{array}{c}\text { Total patients with } \\
1 \text { or more acute/ } \\
\text { relevant events }\end{array}$ \\
\hline & Duane retraction syndrome 1 & 126800 & $18(3.31)$ & 128 & 0 & 0 \\
\hline & Keratoconus & 148300 & $1(0.18)$ & 1 & 0 & 0 \\
\hline & Lattice degeneration of retina & 150500 & $2(0.37)$ & 39 & 0 & 0 \\
\hline & $\begin{array}{l}\text { Macular dystrophy, vitelliform/ } \\
\text { Stargardt disease }\end{array}$ & $\begin{array}{r}153700 / \\
248200\end{array}$ & $10(1.84)$ & 115 & 0 & 0 \\
\hline & Optic atrophy 1 & $\begin{array}{r}165500 / \\
605290\end{array}$ & $4(0.73)$ & 70 & 0 & 0 \\
\hline & Pigmentary retinopathies & $\begin{array}{l}169150 / \\
180100 / \\
215500 / \\
268010\end{array}$ & $14(2.57)$ & 191 & 0 & 0 \\
\hline & $\begin{array}{l}\text { Retinal cone degeneration/ } \\
\text { cone-rod dystrophy } 2\end{array}$ & $\begin{array}{r}180020 / \\
120970\end{array}$ & $8(1.47)$ & 81 & 0 & 0 \\
\hline & Oculocutaneous albinism, type I & 203100 & $1(0.18)$ & 8 & 0 & 0 \\
\hline & Alstrom syndrome & 203800 & $1(0.18)$ & 32 & 1 & 1 \\
\hline & Norrie disease & 310600 & $1(0.18)$ & 10 & 0 & 0 \\
\hline & Kearns-Sayre syndrome & 530000 & $2(0.37)$ & 49 & 7 & 2 \\
\hline & Leber optic atrophy & 535000 & $2(0.37)$ & 5 & 0 & 0 \\
\hline \multirow{7}{*}{$\begin{array}{l}\text { Gastrointestinal } \\
\text { disorders }\end{array}$} & & & $21(3.87 \%)$ & $273(3.8 \%)$ & $6(2.4 \%)$ & $3(14.3 \%)$ \\
\hline & Hirschsprung disease & 142623 & $3(0.55)$ & 32 & 1 & 1 \\
\hline & $\begin{array}{l}\text { Adenomatous polyposis of the } \\
\text { colon }\end{array}$ & 175100 & $1(0.18)$ & 10 & 0 & 0 \\
\hline & Peutz-Jeghers syndrome & 175200 & $1(0.18)$ & 28 & 0 & 0 \\
\hline & Biliary atresia, extrahepatic & 210500 & $1(0.18)$ & 60 & 4 & 1 \\
\hline & Hemochromatosis & 235200 & $14(2.58)$ & 112 & 1 & 1 \\
\hline & Wilson disease & 277900 & $1(0.18)$ & 31 & 0 & 0 \\
\hline \multirow[t]{4}{*}{ Genodermatoses } & & & $17(3.13 \%)$ & $141(2.0 \%)$ & $8(3.3 \%)$ & $6(35.3 \%)$ \\
\hline & Basal cell nevus syndrome & 109400 & $1(0.18)$ & 9 & 2 & 1 \\
\hline & Neurofibromatosis, type I & 162200 & $10(1.84)$ & 87 & 2 & 2 \\
\hline & Tuberous sclerosis & 191100 & $6(1.10)$ & 45 & 4 & 3 \\
\hline \multirow{2}{*}{$\begin{array}{c}\text { Gynecologic } \\
\text { disorders }\end{array}$} & & & $1(0.18 \%)$ & $5(0.07 \%)$ & 0 & 0 \\
\hline & Uterine anomalies & 192000 & $1(0.18)$ & 5 & 0 & 0 \\
\hline \multirow{8}{*}{$\begin{array}{l}\text { Hematologic } \\
\text { disorders }\end{array}$} & & & $118(21.73 \%)$ & $1,744(24.4 \%)$ & $28(11.5 \%)$ & $18(15.3 \%)$ \\
\hline & Antithrombin III deficiency & 107300 & $1(0.18)$ & 19 & 0 & 0 \\
\hline & $\begin{array}{l}\text { Macroglobulinemia, } \\
\text { Waldenstrom }\end{array}$ & 153600 & $1(0.18)$ & 11 & 0 & 0 \\
\hline & $\begin{array}{l}\text { Ovalocytosis, hereditary } \\
\text { hemolytic }\end{array}$ & 166900 & $1(0.18)$ & 27 & 0 & 0 \\
\hline & Pelger-Huet anomaly & 169400 & $1(0.18)$ & 1 & 0 & 0 \\
\hline & Protein C deficiency & 176860 & $3(0.55)$ & 71 & 0 & 0 \\
\hline & Protein S, alpha & 176880 & $2(0.37)$ & 21 & 2 & 1 \\
\hline & & & & & \multicolumn{2}{|c|}{ (Continued on next page) } \\
\hline
\end{tabular}


Table 1

Genetic diagnoses and health events for 2003, continued

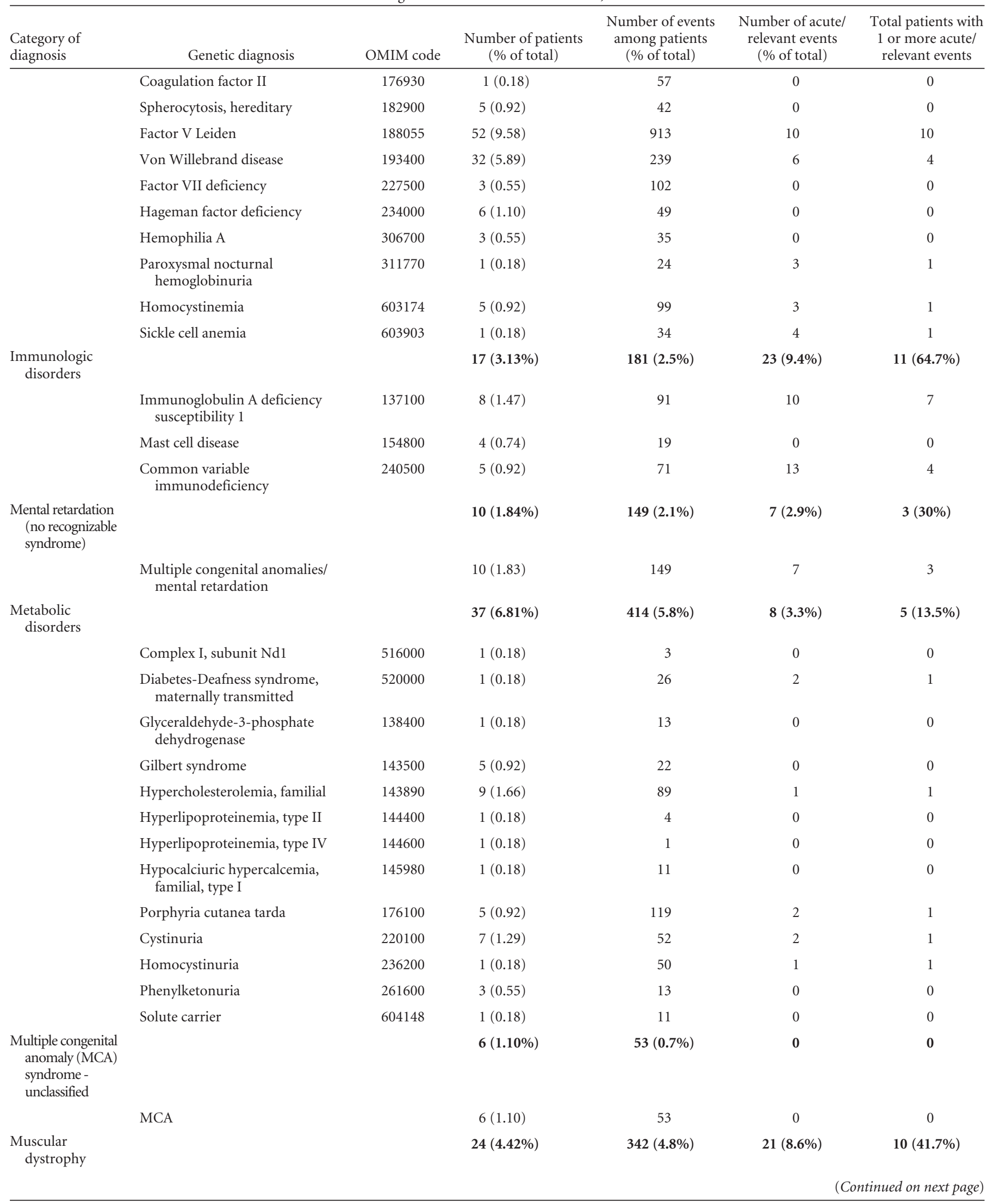


Table 1

Genetic diagnoses and health events for 2003, continued

\begin{tabular}{|c|c|c|c|c|c|c|}
\hline $\begin{array}{l}\text { Category of } \\
\text { diagnosis }\end{array}$ & Genetic diagnosis & OMIM code & $\begin{array}{l}\text { Number of patients } \\
\quad(\% \text { of total })\end{array}$ & $\begin{array}{l}\text { Number of events } \\
\text { among patients } \\
\text { (\% of total) }\end{array}$ & $\begin{array}{l}\text { Number of acute/ } \\
\text { relevant events } \\
\text { (\% of total) }\end{array}$ & $\begin{array}{l}\text { Total patients with } \\
1 \text { or more acute/ } \\
\text { relevant events }\end{array}$ \\
\hline & $\begin{array}{l}\text { Facioscapulohumeral muscular } \\
\text { dystrophy } 1 \mathrm{~A}\end{array}$ & 158900 & $1(0.18)$ & 4 & 0 & 0 \\
\hline & Limb girdle muscular dystrophy & $\begin{array}{c}159000 / \\
253600\end{array}$ & $7(1.28)$ & 83 & 4 & 3 \\
\hline & $\begin{array}{l}\text { Myotonia congenita, Autosomal } \\
\text { dominant }\end{array}$ & 160800 & $3(0.55)$ & 33 & 1 & 1 \\
\hline & Dystrophia myotonia 1 & 160900 & $10(1.84)$ & 160 & 10 & 5 \\
\hline & $\begin{array}{l}\text { Emery-Dreifuss muscular } \\
\text { dystrophy, Autosomal } \\
\text { dominant }\end{array}$ & 181350 & $1(0.18)$ & 13 & 0 & 0 \\
\hline & Dystrophia myotonia 2 & 602668 & $2(0.37)$ & 49 & 6 & 1 \\
\hline \multirow{12}{*}{$\begin{array}{l}\text { Neurologic } \\
\text { conditions }\end{array}$} & & & $27(4.97 \%)$ & $333(4.7 \%)$ & $10(4.1 \%)$ & $8(29.6 \%)$ \\
\hline & Mental retardation, dominant & 156200 & $1(0.18)$ & 8 & 0 & 0 \\
\hline & Neurofibromatosis, type II & 101000 & $1(0.18)$ & 17 & 0 & 0 \\
\hline & $\begin{array}{l}\text { Charcot-Marie-Tooth disease, } \\
\text { type 1A }\end{array}$ & 118220 & $1(0.18)$ & 10 & 1 & 1 \\
\hline & $\begin{array}{l}\text { Arnold Chiari malformation, } \\
\text { type I }\end{array}$ & $\begin{array}{r}118420 / \\
207950\end{array}$ & $6(1.11)$ & 67 & 1 & 1 \\
\hline & Huntington disease & 143100 & $1(0.18)$ & 18 & 1 & 1 \\
\hline & Amyotrophy, Scapuloperoneal & 181400 & $1(0.18)$ & 18 & 0 & 0 \\
\hline & Spina bifida & 182940 & $11(2.03)$ & 157 & 6 & 4 \\
\hline & Syringomyelia & 186700 & $1(0.18)$ & 20 & 1 & 1 \\
\hline & Microcephaly, primary & $\begin{array}{r}251200 / \\
251270\end{array}$ & $2(0.36)$ & 7 & 0 & 0 \\
\hline & Spinal muscular atrophy III & 253400 & $1(0.18)$ & 2 & 0 & 0 \\
\hline & Familial polyneuropathy & & $1(0.18)$ & 9 & 0 & 0 \\
\hline \multirow{4}{*}{$\begin{array}{l}\text { Pulmonary } \\
\text { conditions }\end{array}$} & & & $21(3.87 \%)$ & $270(3.8 \%)$ & $6(2.5 \%)$ & $5(23.8 \%)$ \\
\hline & Protease inhibitor 1 & 107400 & $13(2.39)$ & 176 & 1 & 1 \\
\hline & Cystic fibrosis & 219700 & $5(0.92)$ & 68 & 4 & 3 \\
\hline & Kartagener syndrome & 244400 & $3(0.55)$ & 26 & 1 & 1 \\
\hline \multirow[t]{6}{*}{ Renal conditions } & & & $39(7.18 \%)$ & $592(8.3 \%)$ & $19(7.8 \%)$ & $8(20.5 \%)$ \\
\hline & $\begin{array}{l}\text { Alport syndrome, Autosomal } \\
\text { dominant }\end{array}$ & 104200 & $2(0.37)$ & 26 & 1 & 1 \\
\hline & Adult polycystic kidney disease & 173900 & $33(6.08)$ & 515 & 18 & 7 \\
\hline & Medullary cystic kidney disease 1 & 174000 & $1(0.18)$ & 2 & 0 & 0 \\
\hline & Alport syndrome, X-linked & 301050 & $1(0.18)$ & 1 & 0 & 0 \\
\hline & Renal malformation & & $2(0.37)$ & 48 & 0 & 0 \\
\hline \multirow{7}{*}{$\begin{array}{l}\text { Skeletal dysplasia/ } \\
\quad \text { skeletal } \\
\text { conditions }\end{array}$} & & & $12(2.21 \%)$ & $168(2.4 \%)$ & $9(3.7 \%)$ & $3(25 \%)$ \\
\hline & Achondroplasia & 100800 & $1(0.18)$ & 26 & 3 & 1 \\
\hline & Acrodysostosis & 101800 & $1(0.18)$ & 2 & 0 & 0 \\
\hline & Epiphyseal dysplasia, multiple, 1 & 132400 & $1(0.18)$ & 16 & 0 & 0 \\
\hline & $\begin{array}{l}\text { Fibrodysplasia ossificans } \\
\text { progressiva }\end{array}$ & 135100 & $1(0.18)$ & 1 & 1 & 1 \\
\hline & Hypochondroplasia & 146000 & $2(0.37)$ & 18 & 0 & 0 \\
\hline & & & & & \multicolumn{2}{|c|}{ (Continued on next page) } \\
\hline
\end{tabular}


Table 1

Genetic diagnoses and health events for 2003, continued

\begin{tabular}{|c|c|c|c|c|c|c|}
\hline $\begin{array}{l}\text { Category of } \\
\text { diagnosis }\end{array}$ & Genetic diagnosis & OMIM code & $\begin{array}{l}\text { Number of patients } \\
\text { (\% of total) }\end{array}$ & $\begin{array}{l}\text { Number of events } \\
\text { among patients } \\
\text { (\% of total) }\end{array}$ & $\begin{array}{l}\text { Number of acute/ } \\
\text { relevant events } \\
\text { (\% of total) }\end{array}$ & $\begin{array}{c}\text { Total patients with } \\
1 \text { or more acute/ } \\
\text { relevant events }\end{array}$ \\
\hline & Metachondromatosis & 156250 & $1(0.18)$ & 57 & 5 & 1 \\
\hline & Osteogenesis imperfecta, type I & 166200 & $2(0.37)$ & 21 & 0 & 0 \\
\hline & Roberts syndrome & 268300 & $1(0.18)$ & 3 & 0 & 0 \\
\hline & Polydactyly & 603596 & $1(0.18)$ & 3 & 0 & 0 \\
\hline & Skeletal dysplasia & & $1(0.18)$ & 21 & 0 & 0 \\
\hline \multirow{3}{*}{$\begin{array}{l}\text { Vascular } \\
\quad \text { conditions }\end{array}$} & & & $3(0.55 \%)$ & $60(0.8 \%)$ & 0 & $\mathbf{0}$ \\
\hline & $\begin{array}{l}\text { Hereditary hemorrhagic } \\
\text { telangiectasia }\end{array}$ & 187300 & $2(0.37)$ & 26 & 0 & 0 \\
\hline & AV malformation & & $1(0.18)$ & 34 & 0 & 0 \\
\hline \multirow{11}{*}{$\begin{array}{l}\text { Well-known } \\
\text { non- } \\
\text { chromosomal } \\
\text { conditions }\end{array}$} & & & $16(2.95 \%)$ & $227(3.2 \%)$ & $10(4.1 \%)$ & $7(43.8 \%)$ \\
\hline & Cardiofaciocutaneous syndrome & 115150 & $1(0.18)$ & 3 & 0 & 0 \\
\hline & Sotos syndrome & 117550 & $1(0.18)$ & 27 & 0 & 0 \\
\hline & Holt-Oram syndrome & 142900 & $1(0.18)$ & 19 & 1 & 1 \\
\hline & Klippel-Feil syndrome & 148900 & $2(0.37)$ & 18 & 1 & 1 \\
\hline & $\begin{array}{l}\text { Klippel-Trenaunay-Weber } \\
\text { syndrome }\end{array}$ & 149000 & $3(0.55)$ & 61 & 4 & 3 \\
\hline & Noonan syndrome & 163950 & $3(0.55)$ & 29 & 0 & 0 \\
\hline & VATER association & 192350 & $1(0.18)$ & 3 & 0 & 0 \\
\hline & Von Hippel-Lindau syndrome & 193300 & $1(0.18)$ & 21 & 1 & 1 \\
\hline & Hemihypertrophy & 235000 & $2(0.37)$ & 45 & 3 & 1 \\
\hline & Hypomelanosis of Ito & 300337 & $1(0.18)$ & 1 & 0 & 0 \\
\hline $\begin{array}{l}\text { Totals - All } \\
\text { categories }\end{array}$ & & & $543^{a}$ & 7139 & 244 & 126 \\
\hline
\end{tabular}

${ }^{a}$ This represents 527 unique patients of whom 16 have 2 diagnoses.

their individual risk of presenting with an acute, relevant event was $15 \%$ (calculated by dividing 18 [total patients with 1 or more acute/relevant event] by 118 [total number of patients]).

In addition to chromosomal and hematologic disorders, other categories of genetic conditions contributing substantial proportions of acute relevant health events included connective tissue disorders ( $10.2 \%$ of total) and muscular dystrophies ( $8.6 \%$ of total). On the other hand, other genetic conditions contributed only modestly to the burden of acute relevant health events. For example, the 66 patients with genetic conditions affecting the eyes accounted for only $3.3 \%$ of the total acute relevant events, and their individual risk of such an event was only $4.5 \%$ (calculated by dividing 3 [total patients with 1 or more acute/relevant event] by 66 [total number of patients]).

Table 2 describes the nature of the acute relevant health events identified among the 527 unique patients with underlying genetic conditions. The organ system that was responsible for the presenting problem and the specific diagnosis corre- sponding to the acute visit are indicated. Fifty-three hospital admissions were required for management of some of these acute relevant events (21.7\% of total acute relevant events), and these are summarized in Table 3. Health events related to Down syndrome, followed by adult polycystic kidney disease, sickle cell anemia, and myotonic dystrophy (types 1 and 2) in descending order respectively, accounted for the greatest frequencies of relevant hospital admissions.

The large majority $(34 / 52$ total events $=65 \%)$ of the acute relevant events among patients with underlying chromosomal abnormalities occurred in patients with Down syndrome. Nearly $50 \%$ of the acute relevant events and 3 of 8 hospital admissions among patients with Down syndrome were attributed to immune system dysfunction. Down syndrome accounted for the greatest number of acute relevant events observed in association with immune system defects in all genetic conditions identified. Three osteoporosis-related events, including pathologic fracture and foot pain, were identified in our chart review. A soft-tissue hema- 
Table 2

Description of acute relevant events among the 527 unique patients

Organ system

Genetic diagnosis
Chromosomal and microdeletion
syndromes

Angelman syndrome

Prader-Willi syndrome

Velocardiofacial syndrome

Down syndrome

Klinefelter syndrome

Turner syndrome

3p Monosomy

Connective tissue disorders

Stickler syndrome, type I

Ehlers-Danlos syndrome, type III

Marfan syndrome

Pseudoxanthoma elasticum dominant/ recessive

MASS syndrome

Connective tissue disorder unspecified

\section{Dermatologic}

Ichthyosis, Lamellar, Autosomal dominant form

\section{ENT}

Pendred syndrome

Eye

Alstrom syndrome

Kearns-Sayre syndrome

\section{Gastrointestinal disorders}

Biliary atresia, extrahepatic

Hirschsprung disease

Hemochromatosis

Genodermatoses

Basal cell nevus syndrome
Tremor

$$
\text { - }
$$$$
-
$$

Early dementia, Hematoma, Somulence

$$
\text { Secondary }
$$$$
\text { polycythemia }
$$

CV

$-$

$-$

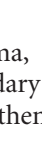

a

\begin{tabular}{cc} 
Derm & Endo \\
\hline & \\
- & - \\
- & - \\
- & - \\
& Goiter, \\
Hypothyroidism, \\
Osteoporosis
\end{tabular}

Endo

ENT

Eye

$$
\text { - } \quad \text { Cellulitis (2) }
$$

-

Dysphagia

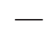

-

-

$-$

$\begin{array}{ccc}- & \begin{array}{c}\text { Chest pain, } \\ \text { Arrhythmia }\end{array} & - \\ - & \text { Coronary infarct } & - \\ - & - & - \\ - & \text { Varicose veins } & \\ & & \\ - & - & \begin{array}{c}\text { Impacted cerumen, } \\ \text { Rash, Cellulitis }\end{array}\end{array}$

-

$$
-
$$$$
\text { - }
$$$$
\text { - }
$$

Otitis media (2)

-

$\begin{array}{ll}- & - \\ - & -\end{array}$

$-$

-

$-$

$$
\text { Diabetes }
$$

Vertigo

Corneal ulcer, Myogenic ptosis 
Table 2

Description of acute relevant events among the 527 unique patients, continued

\begin{tabular}{|c|c|c|c|c|c|c|c|}
\hline \multicolumn{8}{|c|}{ Organ system } \\
\hline GI & GU & Heme & Imm & MS & Psych & Resp & $\begin{array}{l}\text { Total } \\
\text { events }\end{array}$ \\
\hline - & - & - & - & - & - & - & 1 \\
\hline- & - & - & - & Chest pain & Pain obsession & - & 2 \\
\hline- & - & - & - & - & $\begin{array}{l}\text { Depression, Suicide } \\
\text { attempt }\end{array}$ & - & 2 \\
\hline $\begin{array}{l}\text { Cholelithiasis (2), Ulcer, } \\
\text { Ileostomy bleeding, } \\
\text { Crohn disease }\end{array}$ & $\begin{array}{l}\text { Dialysis shunt } \\
\text { dysfunction, UTI }\end{array}$ & - & $\begin{array}{l}\text { Otorrhea, Sinusitis (2), } \\
\text { Bronchiectasis, } \\
\text { Cellulitis (3), URI, } \\
\text { Pneumonia (5), } \\
\text { Mononucleosis, } \\
\text { Ringworm, Otitis } \\
\text { externa }\end{array}$ & $\begin{array}{l}\text { Pathologic } \\
\quad \text { fracture (2) }\end{array}$ & Mood disorder & Sleep apnea & 34 \\
\hline - & Impotence & - & - & - & $\begin{array}{l}\text { Somatization (3), } \\
\text { Depression, } \\
\text { Anxiety (2) }\end{array}$ & - & 9 \\
\hline- & UTI & - & - & Foot pain & - & - & 2 \\
\hline - & - & - & - & - & $\begin{array}{l}\text { Extrapyramidal side } \\
\text { effects }\end{array}$ & - & 2 \\
\hline - & - & - & - & - & - & - & 2 \\
\hline GE reflux & - & - & - & $\begin{array}{l}\text { Hand pain, } \\
\text { Arthralgias, Back } \\
\text { pain (3), Joint } \\
\text { trauma (2), } \\
\text { Intractable pain }\end{array}$ & - & - & 9 \\
\hline - & - & - & - & Chest pain (2) & Depression (2) & - & 6 \\
\hline GERD & - & - & - & - & - & - & 2 \\
\hline Intestinal obstruction & - & - & - & - & - & - & 1 \\
\hline- & - & - & - & $\begin{array}{l}\text { Sprain, Osteoarthritis, } \\
\text { Limb pain (2) }\end{array}$ & - & - & 5 \\
\hline- & - & - & - & - & - & - & 3 \\
\hline - & - & - & - & - & - & - & 1 \\
\hline- & - & - & Cellulitis & - & - & - & 1 \\
\hline GERD & - & - & - & Myopathy, Fall & - & Pneumonia & 7 \\
\hline GI bleed & - & Anemia & $\begin{array}{l}\text { Cellulitis, } \\
\text { Pseudomonal sepsis }\end{array}$ & - & - & - & 4 \\
\hline Irritable colon & - & - & - & - & - & - & 1 \\
\hline - & - & - & - & - & - & - & 1 \\
\hline
\end{tabular}


Table 2

Description of acute relevant events among the 527 unique patients, continued

\begin{tabular}{|c|c|c|c|c|c|c|}
\hline \multirow[b]{2}{*}{ Genetic diagnosis } & \multicolumn{6}{|c|}{ Organ system } \\
\hline & CNS & $\mathrm{CV}$ & Derm & Endo & ENT & Eye \\
\hline Neurofibromatosis, type I & Headache & - & $\begin{array}{l}\text { Necrotic } \\
\text { neurofibroma }\end{array}$ & - & - & - \\
\hline Tuberous sclerosis & $\begin{array}{l}\text { Agitation (2), } \\
\text { Vomiting }\end{array}$ & - & Angiolipoma & - & - & - \\
\hline \multicolumn{7}{|l|}{ Hematologic disorders } \\
\hline Protein S, Alpha & - & Cellulitis (2) & - & - & - & - \\
\hline Factor V Leiden & $\begin{array}{l}\text { Cerebral artery } \\
\text { occlusion, } \\
\text { Left cerebral } \\
\text { infarct }\end{array}$ & $\begin{array}{l}\text { Claudication } \\
\text { pain }(2)\end{array}$ & $\begin{array}{l}\text { Venous } \\
\quad \text { insufficiency }\end{array}$ & - & & - \\
\hline Von Willebrand disease & - & - & - & - & - & - \\
\hline Paroxysmal nocturnal hemoglobinuria & - & - & - & - & - & - \\
\hline Homocystinemia & Dementia & Chest pain & - & - & - & - \\
\hline Sickle cell anemia & - & - & - & - & - & - \\
\hline \multicolumn{7}{|l|}{ Immunologic disorders } \\
\hline $\begin{array}{l}\text { Immunoglobulin A deficiency } \\
\text { susceptibility } 1\end{array}$ & - & - & - & - & - & - \\
\hline Common variable immunodeficiency & - & - & - & - & - & - \\
\hline \multicolumn{7}{|l|}{$\begin{array}{l}\text { Mental retardation (no recognizable } \\
\text { syndrome) }\end{array}$} \\
\hline $\begin{array}{l}\text { Multiple congenital anomalies, Mental } \\
\text { retardation }\end{array}$ & - & - & - & - & - & - \\
\hline \multicolumn{7}{|l|}{ Metabolic disorders } \\
\hline Hypercholesterolemia, familial & - & - & - & - & - & - \\
\hline Porphyria cutanea tarda & - & - & Rash & - & - & - \\
\hline Cystinuria & - & - & - & - & - & - \\
\hline Homocystinuria & - & - & - & - & - & - \\
\hline $\begin{array}{l}\text { Diabetes-deafness syndrome, } \\
\text { maternally transmitted }\end{array}$ & - & - & - & - & - & - \\
\hline \multicolumn{7}{|l|}{ Muscular dystrophy } \\
\hline $\begin{array}{l}\text { Muscular dystrophy, Limb-Girdle, } \\
\text { type 1A }\end{array}$ & - & - & - & - & - & - \\
\hline $\begin{array}{l}\text { Muscular dystrophy, Limb-Girdle, } \\
\text { type } 2 \mathrm{~A}\end{array}$ & - & - & - & - & - & - \\
\hline $\begin{array}{l}\text { Myotonia congenita, autosomal } \\
\text { dominant }\end{array}$ & - & - & - & - & - & - \\
\hline
\end{tabular}


Table 2

Description of acute relevant events among the 527 unique patients, continued

\begin{tabular}{|c|c|c|c|c|c|c|c|}
\hline \multicolumn{8}{|c|}{ Organ system } \\
\hline GI & GU & Heme & Imm & MS & Psych & Resp & $\begin{array}{c}\text { Total } \\
\text { events }\end{array}$ \\
\hline - & - & - & - & - & - & - & 2 \\
\hline - & - & - & - & - & - & - & 2 \\
\hline - & - & - & - & - & - & - & 4 \\
\hline - & - & - & - & - & - & - & 2 \\
\hline - & Threatened abortion & $\begin{array}{l}\text { Hematuria, } \\
\text { Buttock } \\
\text { contusion, } \\
\text { Lower leg } \\
\text { contusion, } \\
\text { Epistaxis }\end{array}$ & - & - & - & - & 10 \\
\hline GI bleed & - & $\begin{array}{c}\text { Preop DDAVP (4), } \\
\text { Metrorrhagia } \\
\text { and Preop } \\
\text { DDAVP }\end{array}$ & - & - & - & - & 6 \\
\hline - & Acute renal failure (2) & $\begin{array}{l}\text { Hemolytic } \\
\text { hemoglobinuria }\end{array}$ & - & - & - & - & 3 \\
\hline GI bleed & - & - & - & - & - & - & 3 \\
\hline - & - & Sickle cell crisis (4) & - & - & - & - & 4 \\
\hline - & - & - & $\begin{array}{c}\text { Cellulitis (3), Sinusitis } \\
\text { (2), Bronchiectasis, } \\
\text { Bronchitis, Acute } \\
\text { pharyngitis (2), } \\
\text { Otitis media }\end{array}$ & - & - & - & 10 \\
\hline Diarrhea & - & - & $\begin{array}{l}\text { Acute sinusitis (5), } \\
\text { Pulmonary fibrosis } \\
\text { (3), Oral candidiasis, } \\
\text { Otitis media }\end{array}$ & - & - & Bronchitis (2) & 13 \\
\hline $\begin{array}{l}\text { Abdominal pain, } \\
\text { Nausea and vomiting }\end{array}$ & $\begin{array}{l}\text { UTI (2), Urine } \\
\text { retention (2) }\end{array}$ & - & - & Limb pain & - & - & 7 \\
\hline - & - & - & - & Chest pain & & & 1 \\
\hline - & - & Fe overload & - & - & - & - & 2 \\
\hline - & Nephrolithiasis (2) & - & - & - & - & - & 2 \\
\hline - & - & - & - & - & $\begin{array}{l}\text { Schizoaffective } \\
\text { disorder }\end{array}$ & - & 1 \\
\hline $\begin{array}{l}\text { Rectal bleed, } \\
\text { Gastroparesis }\end{array}$ & - & - & - & - & - & - & 2 \\
\hline - & - & - & - & Fall & - & $\begin{array}{l}\text { Aspiration } \\
\text { pneumonia }\end{array}$ & 2 \\
\hline - & - & - & - & $\begin{array}{l}\text { Muscle strain, Ankle } \\
\text { sprain }\end{array}$ & - & - & 2 \\
\hline - & - & - & - & Neck pain & - & $\frac{-}{\text { (Continued on nes }}$ & xt page) \\
\hline
\end{tabular}


Table 2

Description of acute relevant events among the 527 unique patients, continued

Organ system

\section{Genetic diagnosis}

Dystrophia myotonia 1

Dystrophia myotonia 2

\section{Neurologic}

Charcot-Marie-Tooth disease, type 1A

Arnold Chiari malformation, type I Neuropathy

Huntington disease

Spina bifida

Syringomyelia

Pulmonary conditions

Protease inhibitor 1

Cystic fibrosis

Kartagener syndrome

Renal conditions

Alport syndrome, autosomal dominant

Adult polycystic kidney disease

\section{Skeletal dysplasia/skeletal conditions}

Achondroplasia

Fibrodysplasia ossificans progressiva

Metachondromatosis

Well-known non-chromosomal conditions

Holt-Oram syndrome

Klippel-Feil syndrome

Klippel-Trenaunay-Weber syndrome

Von Hippel-Lindau syndrome

Hemihypertrophy

Total

12

Atrial fibrillation

Derm

Endo

ENT

Eye

$-$

$-$

$-$

$-$

-
Decubitus ulcer

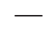

$-$

-

$-$

$-$ failure
- Congestive heart Skin ulcer
Acute sinusitis

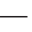

$-$
$-$

$-$ 
Table 2

Description of acute relevant events among the 527 unique patients, continued

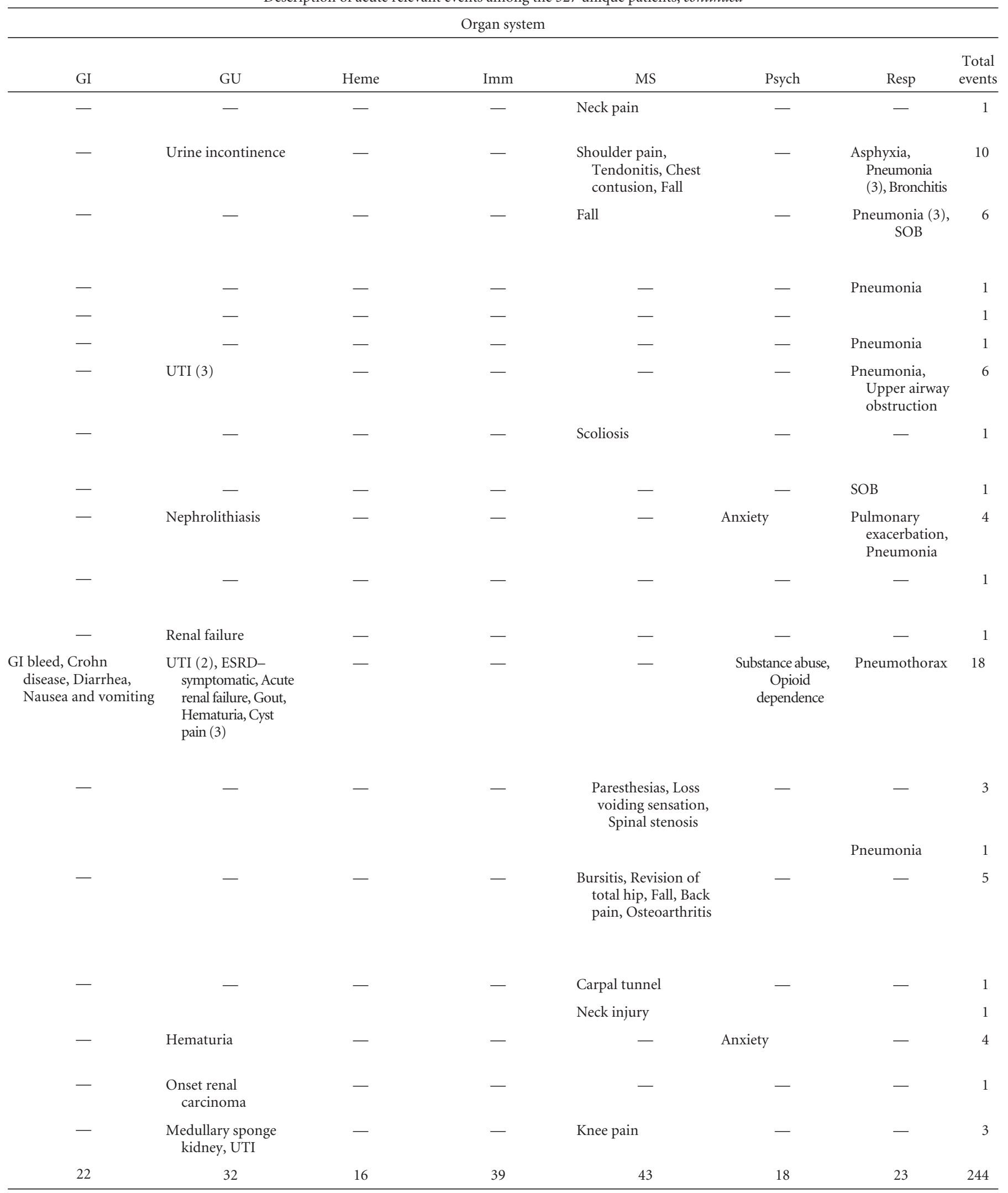

CNS, central nervous system; CV, cardiovascular; Derm, dermatology; Endo, endocrinology; ENT, ear, nose, throat; ESRD, end stage renal disease; GE, gastroesophageal; GERD, gastroesophageal reflux disease; GI, gastrointestinal; GU, genitourinary; Heme, hematology; Imm, immunology; MS, musculoskeletal; Psycho, psychology; Resp, respiratory; SOB, shortness of breath; URI, upper respiratory infection; UTI, urinary tract infection. 
Table 3

Hospital admissions (53) for acute relevant events

\begin{tabular}{|c|c|}
\hline Name of genetic condition & Emergency department diagnosis \\
\hline Down syndrome & $\begin{array}{l}\text { Cholelithiasis, goiter, mononucleosis, sleep } \\
\text { apnea, ulcer, cellulitis, somulence, } \\
\text { pneumonia }\end{array}$ \\
\hline $\begin{array}{l}\text { Multiple congenital } \\
\text { anomalies, Mental } \\
\text { retardation }\end{array}$ & Nausea and vomiting \\
\hline Achondroplasia & Spinal stenosis \\
\hline $\begin{array}{l}\text { Alport syndrome, } \\
\text { autosomal dominant }\end{array}$ & Renal failure \\
\hline Alstrom syndrome & Cellulitis \\
\hline $\begin{array}{l}\text { Biliary atresia, } \\
\text { extrahepatic }\end{array}$ & Cellulitis, Pseudomonal sepsis, GI bleed \\
\hline $\begin{array}{l}\text { Common variable } \\
\text { immunodeficiency }\end{array}$ & Pulmonary fibrosis \\
\hline Dystrophia myotonia 1 & Pneumonia \\
\hline Dystrophia myotonia 2 & Atrial fibrillation, pneumonia (2) \\
\hline $\begin{array}{l}\text { Ehlers-Danlos syndrome, } \\
\text { type III }\end{array}$ & Back pain, joint trauma \\
\hline Homocystinemia & Chest pain, GI bleed \\
\hline Homocystinuria & Schizoaffective disorder \\
\hline Huntington disease & Pneumonia \\
\hline Kearns-Sayre syndrome & Pneumonia \\
\hline Klippel-Feil syndrome & Neck injury \\
\hline Marfan syndrome & Chest pain, depression \\
\hline MASS syndrome & Intestinal obstruction \\
\hline Metachondromatosis & Bursitis \\
\hline $\begin{array}{l}\text { Muscular dystrophy, } \\
\text { Limb-girdle, type } 1 \mathrm{~A}\end{array}$ & Aspiration pneumonia \\
\hline $\begin{array}{l}\text { Paroxysmal nocturnal } \\
\text { hemoglobinuria }\end{array}$ & $\begin{array}{l}\text { Hemolytic hemoglobinuria, acute renal } \\
\text { failure (2) }\end{array}$ \\
\hline $\begin{array}{l}\text { Adult polycystic kidney } \\
\text { disease }\end{array}$ & $\begin{array}{l}\text { Acute renal failure, GI bleed, } \\
\text { pneumothorax, opioid dependence, } \\
\text { substance abuse }\end{array}$ \\
\hline $\begin{array}{l}\text { Pseudoxanthoma } \\
\text { elasticum, autosomal } \\
\text { recessive }\end{array}$ & Coronary infarct \\
\hline Sickle cell anemia & Sickle cell crisis (4) \\
\hline Spina bifida & Pneumonia, upper airway obstruction \\
\hline Factor V Leiden & $\begin{array}{l}\text { Cerebral artery occlusion, left cerebral } \\
\text { infarct }\end{array}$ \\
\hline Tuberous sclerosis & Agitation \\
\hline Velocardiofacial syndrome & Suicide attempt \\
\hline Von Willebrand disease & GI bleed \\
\hline
\end{tabular}

toma occurred in one patient with Down syndrome who was on anticoagulant therapy following heart surgery.

One patient with Klinefelter syndrome had two episodes of cellulitis secondary to stasis dermatitis. Five patients with von
Willebrand disease required vasopressin (DDAVP) prior to surgical procedures. A gastrointestinal bleed was observed in one patient with adult polycystic kidney disease and Crohn disease.

Chromosome disorders were associated with 10 of 18 acute relevant psychiatric health events observed in the 2003 calendar year. Six of these relevant psychiatric events occurred in patients with Klinefelter syndrome and the most serious event, a suicide attempt that required hospital admission, was reported in a patient with velocardiofacial syndrome.

Twenty-three of 43 acute relevant events involving the musculoskeletal system were related to underlying connective tissue disorders ${ }^{14}$ and muscular dystrophies. ${ }^{9}$ There were eight events associated with Ehlers Danlos syndrome type III, followed by myotonic dystrophy types I and II with five musculoskeletal events.

Fourteen of 23 acute relevant events involving the respiratory system were related to muscular dystrophies and neurologic conditions. Myotonic dystrophy types I and II accounted for nine acute relevant respiratory events.

Acute relevant events associated with underlying hematologic genetic disorders involved primarily the hematologic, genitourinary, and central nervous system. The greatest proportion of acute relevant events ( 10 of 28 ) were associated with Factor V Leiden deficiency.

\section{DISCUSSION}

Through a two-tiered screening approach utilizing computer based diagnostic coding and manual medical record review, we identified a cohort of 591 adult patients with welldefined single gene, chromosomal, and selected multifactorial conditions compromising just over $1 \%$ of a geographicallydefined rural population in central Wisconsin. We also manually reviewed a total of 7,139 outpatient visits among the 527 patients with health visits during calendar year 2003 in order to delineate acute health events which were of relevance to each patient's particular disease. We discovered that chromosomal and microdeletion syndromes (21.3\%), hematologic disorders $(11.5 \%)$, connective tissue disorders (10.2\%), and muscular dystrophies $(8.6 \%)$ together accounted for more than half of the acute relevant health events occurring among adults in this study cohort. We also learned that immunologic conditions, chromosomal disorders, muscular dystrophy, genodermatoses, and several well-known non-chromosomal disorders conveyed a fairly high risk (35-65\%) of presenting with an acute relevant event in the course of a calendar year. To our knowledge, this represents the first study of acute health events in a large population-based cohort of adult patients with a broad array of genetic diseases. Our purpose was to identify patients with underlying genetic conditions who are vulnerable to acute health problems so as to provide information for patients, families, and health care providers about the natural history of genetic conditions, and which can support development of appropriate prevention strategies. 
A substantial proportion (22.1\%) of acute and relevant health events occurred at a visit in the primary care provider or subspecialist's office practice as opposed to an urgent care setting, highlighting a justification for continued educational efforts and awareness among both primary and subspecialty care providers of the natural history of adult genetic conditions and their associated morbidities. This study reports on acute health events in patients with known genetic disease. However, these same events could also represent the initial presenting symptoms for patients with undiagnosed genetic conditions. There are currently relatively few genetic conditions in which clinical outcomes are known in adulthood, making it difficult to provide health monitoring and treatment.

These data have relevance for the clinical geneticist who often serves as an important liaison between the patient and adult primary care provider. Providing the patient and family with a health management plan and insight into different health events which are associated with a given genetic condition may help reduce morbidity and mortality and promote earlier recognition of acute complications of a given condition and/or facilitate diagnosis if a relevant acute event occurs in a previously undiagnosed patient. Although a family with a newly diagnosed child with Down syndrome may see a geneticist to discuss associated medical issues, some adult patients with Down syndrome may not be seen by a clinical geneticist after this initial visit. There is little opportunity for the geneticist to provide education to the family about the adult complications of Down syndrome, some of which are highlighted in Table 2. The clinical geneticist can help to facilitate this process through periodic follow-up in adult patients with selected genetic diagnoses and providing the family and primary care provider a letter summarizing recommended health maintenance guidelines.

Psychiatric problems occurred in a variety of chromosomal disorders, and were particularly more frequent in Klinefelter syndrome as compared to Down syndrome. Previous literature gives little evidence of acute psychiatric events in Klinefelter syndrome although psychosocial adjustment problems among males with Klinefelter syndrome appear to be increased. ${ }^{17} \mathrm{An}$ evaluation of 12 boys with prenatally diagnosed Klinefelter syndrome indicated significant delays in expressive language (91.7\%) and emotional disturbances in 50\% including withdrawal, depression, anxiety, social problems, delinquency, and aggressive behavior. ${ }^{18}$ Reporting of psychiatric illness in adults with Klinefelter syndrome has been limited. Periodic behavior assessment and monitoring may help to avoid serious psychiatric events. Another surprisingly significant problem was narcotic dependence among patients with adult polycystic kidney disease. This is another area in which prevention strategies and intervention could be of benefit. We have not been able to find any reported studies that address this health issue.

As children with genetic conditions enter adulthood, adequate health coverage is an important issue that needs to be addressed. Longevity has improved for many genetic conditions including Down syndrome, cystic fibrosis, and Marfan syndrome, and practitioners are facing health concerns of adult patients with these, as well as other genetic conditions.
Improvement in our ability to track and define health outcomes in patients with genetic conditions is important in order to enable the medical community to learn what health problems pose a significant risk for a patient with a specific genetic condition. For example, immune defects have been reported in patients with Down syndrome. ${ }^{19,20}$ Recent evidence indicates that children with Down syndrome have a 30\% increased risk of fatality due to sepsis as compared to patients without Down syndrome. ${ }^{20}$ Further epidemiologic studies for adults with Down syndrome are needed to more completely characterize sepsis fatality risks in adult Down syndrome patients. Osteoporosis has previously been identified to be a health problem in adults with Down syndrome. ${ }^{21}$ As survival of Down syndrome patients increases, pathologic fractures may become an important and potentially preventable cause of morbidity.

The relevance of two acute relevant health events reported for adult polycystic kidney disease patients in Table 2 requires clarification. Crohn disease was previously reported in a patient who underwent renal transplantation for adult polycystic kidney disease although the mechanism of association is not entirely clear. ${ }^{22}$ Pneumothorax and cystic lungs have also been previously reported in adult polycystic kidney disease. ${ }^{23-25}$

It is noteworthy that greater than half of the acute relevant musculoskeletal events were due to connective tissue protein abnormalities and neuromuscular abnormalities. These conditions can render an affected individual more susceptible to loss of balance, sprains, and falls.

As applications of the Human Genome Project provide more information about the genetic etiologies of disease, genetic markers will be defined for an increasing number of clinical conditions. Through the use of comparative genomic hybridization, new microduplication and microdeletion syndromes are being defined. Our long-term knowledge of these conditions is far more limited than many of the clinical conditions that we view as common, such as Turner syndrome. Additional efforts are necessary to track these conditions and obtain information about associated health risks.

Although this study was population-based and followed the acute health events of a cohort of patients with well-defined genetic conditions over one year, there are several limitations. Our study was limited to follow-up of individuals with selected genetic conditions who reside in the MESA population. Many genetic conditions were not studied because they were not discovered within the underlying MESA population. For some conditions, the ICD-9 codes selected as a screening tool may not capture all patients with those conditions. Also, we did not follow individuals with commonly occurring multifactorial conditions, such as hypertension, asthma, or diabetes. Had we used a broader array of genetic conditions, our sample size would have been much larger. Since we performed follow-up for one year, the number of health events was limited and not all inclusive of the various possibilities for acute health events associated with a particular syndrome. It is possible that acute relevant health events that did not occur within the Marshfield Clinic system were not identified, although previous validation studies suggest such events are quite uncommon. Assessment 
of relevance was difficult for some events and was based on the known pathophysiology of the genetic condition. We may have excluded some events that were causally related but unsupported by current knowledge. Since the group of subjects with any given diagnosis was reviewed by the same geneticist, any unusual pattern would have been recognized, even if it was not a previously recognized complication. Our study only focused on acute health events with a given genetic condition. We did not include relevant non-acute health events for a particular genetic condition. We anticipate that future studies can expand on our initial study and complement our findings, and address the limitations that we have identified.

For instance, additional studies could focus more closely on health outcomes in a specific issue such as psychiatric illness in adults with Klinefelter syndrome, opioid dependency in adult polycystic kidney disease, or related connective tissue problems in adult polycystic kidney disease. These efforts can include establishment of a national database that would include multicenter pooling of health event data for genetic diseases. A recent study ${ }^{26}$ surveying family practice physicians regarding genetic issues encountered in practice indicated that $35 \%$ of those physicians surveyed addressed conditions with abnormal numbers of chromosomes. Twenty-eight percent of those physicians surveyed addressed conditions with congenital abnormalities, $27 \%$ of those physicians surveyed addressed conditions with mental retardation, $25 \%$ of those physicians surveyed addressed conditions with neurofibromatosis, $16 \%$ of those physicians surveyed addressed conditions with Marfan syndrome, and $12 \%$ of those physicians surveyed addressed conditions with phenylketonuria and metabolic disorders. These data support the view that the range of genetic problems encountered in adult practice is significant and information regarding adult complications of genetic disease could be beneficial for optimal case management.

In summary we have utilized a population-based cohort to identify acute health events in a cohort of adult patients with genetic disease. Ongoing educational efforts for primary care providers are necessary in order to optimize diagnosis and care for patients with genetic disorders. More widespread efforts are needed in order to help obtain long-term follow-up data on adults with genetic conditions in order to help obtain natural history data, guide preventative health care strategies, and address the economic impact on adult patients with genetic diseases. These efforts have the potential to improve transitioning for adult diseases from adolescence to adulthood.

\section{ACKNOWLEDGMENTS}

The authors thank Sandra Strey and Sonia Stratman for their assistance in review of medical records and Jamie Elliot Steward for database management. We also thank Marshfield Clinic Research Foundation for its support through the assis- tance of Ingrid Glurich, $\mathrm{PhD}$, for her advice, Cathy McCarty, $\mathrm{PhD}$, for her assistance in study design, and Linda Weis and Alice Stargardt in the preparation of this manuscript.

\section{References}

1. Rimoin DL, Connor JM, Pyeritz RE, Korf BR. Nature and frequency of genetic disease. In: Emery and Rimoin's principles and practice of medical genetics. Vol 1. 4th ed. Rimoin DL, Connor JM, Pyeritz RE, Korf BR, editors. New York: Churchill Livingstone;2002:55-59.

2. Childs B, Miller S, Bearn A. Gene mutations as a cause of human disease. In: Sutton HE, Harris MI, editors. Mutagenic effects of environmental contaminants. New York: Academic Press; 1972:3-14.

3. Day N, Holmes LB. The incidence of genetic disease in a University hospital population. Am J Hum Genet 1973;25:237-246.

4. Scriver CR, Neal JL, Saginur R, Clow A. The frequency of genetic disease and congenital malformation among patients in a pediatric hospital. Can Med Assoc J 1973; 108:1111-1115.

5. Hall JG, Powers EK, Mcllvaine RT, Ean VH. The frequency and financial burden of genetic disease in a pediatric hospital. Am J Med Genet 1978;1:417-436.

6. FitzPatrick DR, Skeoch CH, Tolmie JL. Genetic aspects of admissions to a paediatric intensive care unit. Arch Dis Child 1991;66:639-641.

7. Yoon PW, Olney RS, Khoury MJ, Sappenfield WM, et al. Contribution of birth defects and genetic diseases to pediatric hospitalizations. A population-based study. Arch Pediatr Adolesc Med 1997;151:1096-1103.

8. Hall JG. The impact of birth defects and genetic diseases. Arch Pediatr Adolesc Med 1997; 151:1082-1083.

9. McCandless SE, Brunger JW, Cassidy SB. The burden of genetic disease on inpatient care in a children's hospital. Am J Hum Genet 2004;74:121-127.

10. Kumar P, Radhakrishnan J, Chowdhary MA, Giampietro PF. Prevalence and patterns of presentation of genetic disorders in a pediatric emergency department. Mayo Clin Proc 2001;76:777-783.

11. DeStefano F, Eaker ED, Broste SK, Nordstrom DL, et al. Epidemiologic research in an integrated regional medical care system: the Marshfield Epidemiologic Study Area. J Clin Epidemiol 1996;49:643-652.

12. Greenlee RT. Measuring disease frequency in the Marshfield Epidemiologic Study Area (MESA). Clin Med Res 2003;1:273-280.

13. Jones KL. Smith's recognizable patterns of human malformation. $6^{\text {th }}$ ed. Philadelphia, PA: WB Saunders Co; 2006.

14. Scriver CR, Beaudet AL, Sly WS, Valle D, editors. The metabolic and molecular basis of inherited disease. $8^{\text {th }}$ ed. New York: McGraw-Hill; 2001.

15. OMIM ${ }^{\mathrm{TM}}$ Online Mendelian Inheritance in Man. Available at: http://www.ncbi.nlm nih.gov/entrez/query.fcgi?db=OMIM. Accessed on January 15, 2006.

16. University of Washington, Seattle. GeneTests. Located at: http://www.genetests.org/. Accessed on January 15, 2006.

17. Robinson A, Bender BG, Linden MG. Summary of clinical findings in children and young adults with sex chromosome anomalies. Birth Defects Orig Artic Ser 1990;26: 225-228.

18. Meeus G, Steyaert J, Fryns JP. A follow-up study on 12 prenatally diagnosed boys with Klinefelter syndrome. Genet Couns 2005;16:249-256.

19. Ugazio AG, Maccario R, Notarangelo LD, Burgio GR. Immunology of Down syndrome: a review. Am J Med Genet Suppl 1990;7:204-212.

20. Garrison MM, Jeffries H, Christakis DA. Risk of death for children with Down syndrome and sepsis. J Pediatr 2005;147:748-752.

21. van Allen MI, Fung J, Jurenka SB. Health care concerns and guidelines for adults with Down syndrome. Am J Med Genet 1999;89:100-110.

22. Riley TR, Schoen RE, Lee RG, Rakela J. A case series of transplant recipients who despite immunosuppression developed inflammatory bowel disease. Am J Gastroenterol 1997;92 (2):279-282.

23. Kornprat $\mathrm{P}$, Cerwenka H, Bacher H, El-Shabrawi A, et al. Surgical therapy options in polycystic liver disease. Wien Klin Wochenschr. 2005;117:215-218.

24. Pinto MA, Juliano RV, Tobias-Machado M, Borrelli M, et al. Hand-assisted bilateral nephrectomy in a patient with adult polycystic kidney disease. Sao Paulo Med J 2002;120:189-191.

25. Gatalica Z, Schwarting R, Petersen RO. Renal cell carcinoma in the presence of adult polycystic kidney disease. Urology 1994;43:102-105.

26. Acheson LS, Stange KC, Zyzanski S. Clinical genetics issues encountered by family physicians. Genet Med 2005;7:501-508. 